\title{
Synergistic effect of ursodeoxycholic acid on the antitumor activity of sorafenib in hepatocellular carcinoma cells via modulation of STAT3 and ERK
}

\author{
SEULKI LEE, YOUNG YOUN CHO, EUN JU CHO, SU JONG YU, JEONG-HOON LEE, \\ JUNG-HWAN YOON and YOON JUN KIM \\ Department of Internal Medicine and Liver Research Institute, \\ Seoul National University College of Medicine, Seoul 110-799, Republic of Korea
}

Received February 9, 2018; Accepted July 18, 2018

DOI: $10.3892 / \mathrm{jjmm} .2018 .3807$

\begin{abstract}
Sorafenib has been approved for the treatment of advanced stage hepatocellular carcinoma but has limited efficacy. Ursodeoxycholic acid exerts cytoprotective activities in hepatocytes and is believed to suppress tumorigenesis through cell cycle arrest and induction of apoptosis. The present study examined whether co-treatment with ursodeoxycholic acid has a synergistic effect on the antitumor activity of sorafenib in hepatocellular carcinoma cells. Notably, co-treatment with both agents more effectively inhibited cell proliferation than sorafenib or ursodeoxycholic acid alone. Furthermore, co-treatment inhibited the phosphorylation of signal transducer and activator of transcription 3 (STAT3) and activated extracellular signal-regulated kinase (ERK), a mitogen-activated protein kinase, accompanied by excessive intracellular reactive oxygen species generation in hepatocellular carcinoma cells. Thus, chemotherapy with sorafenib and ursodeoxycholic combination may be efficacious in hepatocellular carcinoma by inhibiting cell proliferation and inducing apoptosis through reactive oxygen species-dependent activation of ERK and dephosphorylation of STAT3. The present findings may
\end{abstract}

Correspondence to: Professor Yoon Jun Kim, Department of Internal Medicine and Liver Research Institute, Seoul National University College of Medicine, 103 Daehak Road, Seoul 110-799, Republic of Korea

E-mail: yoonjun@snu.ac.kr

Abbreviations: CDK, cyclin-dependent kinase; ERK, extracellular signal-regulated kinase; FBS, fetal bovine serum; HCC, hepatocellular carcinoma; HRP, horseradish peroxide; KCLB, Korean Cell Line Bank; MAPK, mitogen-activated protein kinase; MEK, MAPK kinase; NAC, N-acetyl cysteine; PI, propidium iodide; ROS, reactive oxygen species; STAT3, signal transducer and activator of transcription 3; TBST, Tris-buffered saline/Tween-20; UDCA, ursodeoxycholic acid

Key words: sorafenib, ursodeoxycholic acid, hepatocellular carcinoma, antitumor activity, reactive oxygen species-dependent activation represent a promising therapeutic strategy for patients with advanced hepatocellular carcinoma.

\section{Introduction}

Hepatocellular carcinoma (HCC) is the most common malignant neoplasm of the liver and the third leading cause of cancer-related mortality worldwide (1). Although curative treatments, such as liver transplantation and surgical resection, are available to some patients with $\mathrm{HCC}$, most patients are often diagnosed at an advanced stage, which is not suitable for curative treatment (2). The prognosis of patients with advanced HCC remains poor, and the therapeutic options available to these patients are limited (3).

Sorafenib, a multikinase inhibitor, is the first clinically approved targeted chemotherapy for HCC $(4,5)$. It suppresses tumor cell proliferation by targeting Raf-1 proto-oncogene, vascularendothelial growth factor receptor, and platelet-derived growth factor receptor signaling pathways (6). In patients with advanced HCC, sorafenib has proven beneficial to survival; however, its main limitation is its low objective response rates. Furthermore, sorafenib therapy is accompanied by significant adverse effects including dermatological, digestive, and cardiovascular toxicities (7). The adverse effects can be managed by sorafenib dose reduction. Therefore, it is necessary to investigate combination treatments with sorafenib to enhance its anticancer effects and reduce its toxicity through dose reduction. To overcome these unmet needs of sorafenib therapy, co-treatment with other chemotherapeutic agents has been previously evaluated (8-10).

Bile acids synthesized from cholesterol in the liver are necessary for the digestion and absorption of lipids; however, elevated hydrophobic bile acid concentrations are associated with pathological activities and promote the development of liver cirrhosis (11). Unlike hydrophobic bile acids, ursodeoxycholic acid (UDCA), a hydrophilic acid, relieves cholestatic liver disease by exerting cytoprotective activities in hepatocytes, and it has been suggested to suppress tumorigenesis through cell cycle arrest and induction of apoptosis $(12,13)$. Although the antitumor role of UDCA in HCC has been investigated, the specific molecular mechanisms are not yet 
fully understood. UDCA is often administered to patients with $\mathrm{HCC}$ in clinical practice and has minimal side effects. In addition, the mechanism of the antitumor effect of UDCA differs from that of sorafenib.

Pro-oxidant or reactive oxygen species (ROS) activity has been demonstrated to contribute to cytotoxicity and subsequent induction of apoptosis (14). Extracellular signal-regulated kinase (ERK), part of the mitogen-activated protein kinase (MAPK) superfamily, has been reported to promote apoptosis in response to external stimuli, such as ROS (15). In addition, signal transducer and activator of transcription 3 (STAT3) is primarily activated by interleukin-6 (IL-6) receptor-associated Janus kinases; however, several studies have suggested that STAT3 is influenced by conditions such as increased pro-oxidant levels that subsequently lead to decreased tumor growth and metastasis $(16,17)$. STAT3 regulates the transcriptional activation of anti-apoptotic genes, such as cyclins and cyclin-dependent kinases (CDKs) (18). To date, investigations of STAT3 function have focused on cancer therapy.

In the present study, it was hypothesized that co-treatment with UDCA would enhance the antitumor efficacy of sorafenib in HCC cells. Thus, the effect of sorafenib and UDCA co-treatment and their underlying molecular mechanisms were investigated in HCC cells.

\section{Materials and methods}

Cell culture and drug treatment. Huh-BAT and HepG2 human HCC cells were purchased from the Korean Cell Line Bank (Seoul, Korea). Cells were maintained in continuous culture in Dulbecco's modified Eagle's medium (Thermo Fisher Scientific, Inc., Waltham, MA, USA) supplemented with $10 \%$ fetal bovine serum (FBS; Thermo Fisher Scientific, Inc.), penicillin $(100 \mathrm{U} / \mathrm{ml})$, and streptomycin $(100 \mathrm{mg} / \mathrm{ml})$. For the experiments, the cells were seeded in tissue culture dishes at $37^{\circ} \mathrm{C}$ in a $5 \% \mathrm{CO}_{2}$ humidified incubator. They were cultured in fresh medium containing $10 \%$ FBS for $24 \mathrm{~h}$ and then treated with different concentrations of UDCA (Sigma-Aldrich; Merck KGaA, Darmstadt, Germany), sorafenib (LC Laboratories, Woburn, MA, USA), N-acetyl cysteine (NAC; Sigma-Aldrich) or U0126 (Cell Signaling Technology, Inc., Danvers, MA, USA). Cells cultured in medium with $10 \%$ FBS, without any additional treatments, were used as controls.

Cell viability assay. Cells were plated in 96-well plates at a density of 5,000 cells/well and 1 day later they were incubated with sorafenib and UDCA for $48 \mathrm{~h}$. Then, the cells were incubated with $0.5 \mathrm{mg} / \mathrm{ml} \mathrm{3-(4,5-dimethylthiazol-2-yl)-2,5-}$ diphenyltetrazolium bromide (MTT; Amresco Inc., Solon, $\mathrm{OH}$, USA) at $37^{\circ} \mathrm{C}$ for $3 \mathrm{~h}$. Following removal of the MTT solution, dimethyl sulfoxide was added to each well with mixing for $15 \mathrm{~min}$, and then the absorbance was detected at $540 \mathrm{~nm}$ using a plate reader.

Annexin V/propidium iodide (PI) apoptosis assay. Annexin V/PI staining was conducted to determine the $\%$ of apoptotic cells in the total cell population. Following sorafenib and UDCA treatment, cells were collected and resuspended in binding buffer (BD Pharmingen; BD Biosciences, San Jose, CA, USA). The cells were then incubated with $10 \mu \mathrm{g} / \mathrm{ml}$ PI and Annexin V
(BD Biosciences), and the fluorescence intensity was determined using a FACSCalibur flow cytometer and BD CellQuest ${ }^{\mathrm{TM}}$ Pro software version 5.2.1 (BD Biosciences).

Immunofluorescence assay. Cells were grown on coverslips in 12-well culture plates and then exposed to UDCA and sorafenib for $24 \mathrm{~h}$, followed by IL- 6 for $30 \mathrm{~min}$. Following fixation with $4 \%$ paraformaldehyde for $20 \mathrm{~min}$ at room temperature, the cells were permeabilized with $100 \%$ methanol at $-20^{\circ} \mathrm{C}$, blocked with $10 \%$ normal goat serum (Vector Laboratories, Ltd., Peterborough, UK) for $1 \mathrm{~h}$ at room temperature $\left(23.8 \pm 6.3^{\circ} \mathrm{C}\right)$, incubated with phosphorylated (p)-STAT3 antibody (1:50; cat. no. 4113; Cell Signaling Technology, Inc.) at $4^{\circ} \mathrm{C}$ overnight, and then reacted with Alexa Fluor 488-conjugated secondary antibody (1:50; cat. no. ab150113; Abcam, Cambridge, UK). for $1 \mathrm{~h}$ at room temperature $\left(23.8 \pm 6.3^{\circ} \mathrm{C}\right)$. The cell nuclei were stained with 4',6-diamidino-2-phenylindole, and the cells were observed using a confocal microscope.

Measurement of ROS production. ROS levels were measured using a cellular ROS detection assay kit (Abcam). For fluorescence microscopy, cells were seeded in 12-well plates, exposed to UDCA or sorafenib for $24 \mathrm{~h}$, and then labeled with an oxidative stress detection reagent (green color) for $2 \mathrm{~h}$, according to the manufacturer's instructions. The fluorescence intensity was then measured using a confocal microscope with Leica Application Suite X (Leica Microsystems GmbH, Wetzlar, Germany). To quantify ROS levels, cells were seeded onto 96-well black plates and then stained with ROS red dye working solution for $1 \mathrm{~h}$ at $37^{\circ} \mathrm{C}$ in a $5 \% \mathrm{CO}_{2}$ humidified incubator. Cells were treated with UDCA or sorafenib for $30 \mathrm{~min}$ and were then analyzed using a fluorescent microplate reader (Molecular Devices, Sunnyvale, CA, USA).

Western blot analysis. Huh-BAT and HepG2 cells were lysed in radioimmunoprecipitation assay buffer (Cell Signaling Technology, Inc.). The protein concentration was determined using BCA assay (Thermo Fisher Scientific, Inc.). The protein samples $(25 \mu \mathrm{g})$ were separated using $8-12 \%$ sodium dodecyl sulfate-polyacrylamide gel electrophoresis and then transferred onto polyvinylidene fluoride membranes (Millipore, Billerica, MA, USA). After blocking with 5\% skim milk in Tris-buffered saline/0.1\% Tween-20 (TBST) for $1 \mathrm{~h}$, the membrane was incubated overnight at $4^{\circ} \mathrm{C}$ with $1: 1,000$ dilutions of primary antibodies against the following proteins: $\beta$-actin (cat. no. sc-47778; Santa Cruz Biotechnology, Inc., Dallas, TX, USA), STAT3, p-STAT3 (Tyr705; cat. no. 4113), ERK (cat. no. 4695s), p-ERK (cat. no. 4377), caspase-9 (cat. no. 9580), cleaved caspase-9 (cat. no. 7237), caspase-3 (cat. no. 9665), and cleaved caspase-3 (cat. no. 9664) (all from Cell Signaling Technology, Inc.). Then, the membranes were incubated with 1:2,500 dilutions of either goat anti-rabbit horseradish peroxidase (HRP)-conjugated secondary antibody (cat. no. sc-2357; Santa Cruz Biotechnology, Inc.) or goat anti-mouse HRP-conjugated secondary antibody (cat. no. 31430; Thermo Fisher Scientific, Inc.) in TBST for $1 \mathrm{~h}$ at room temperature. The blots were developed using enhanced chemiluminescence detection reagents (Promega Corporation, Madison WI, USA). All bands were quantified via densitometry using ImageJ software version 1.51 (National Institutes of 

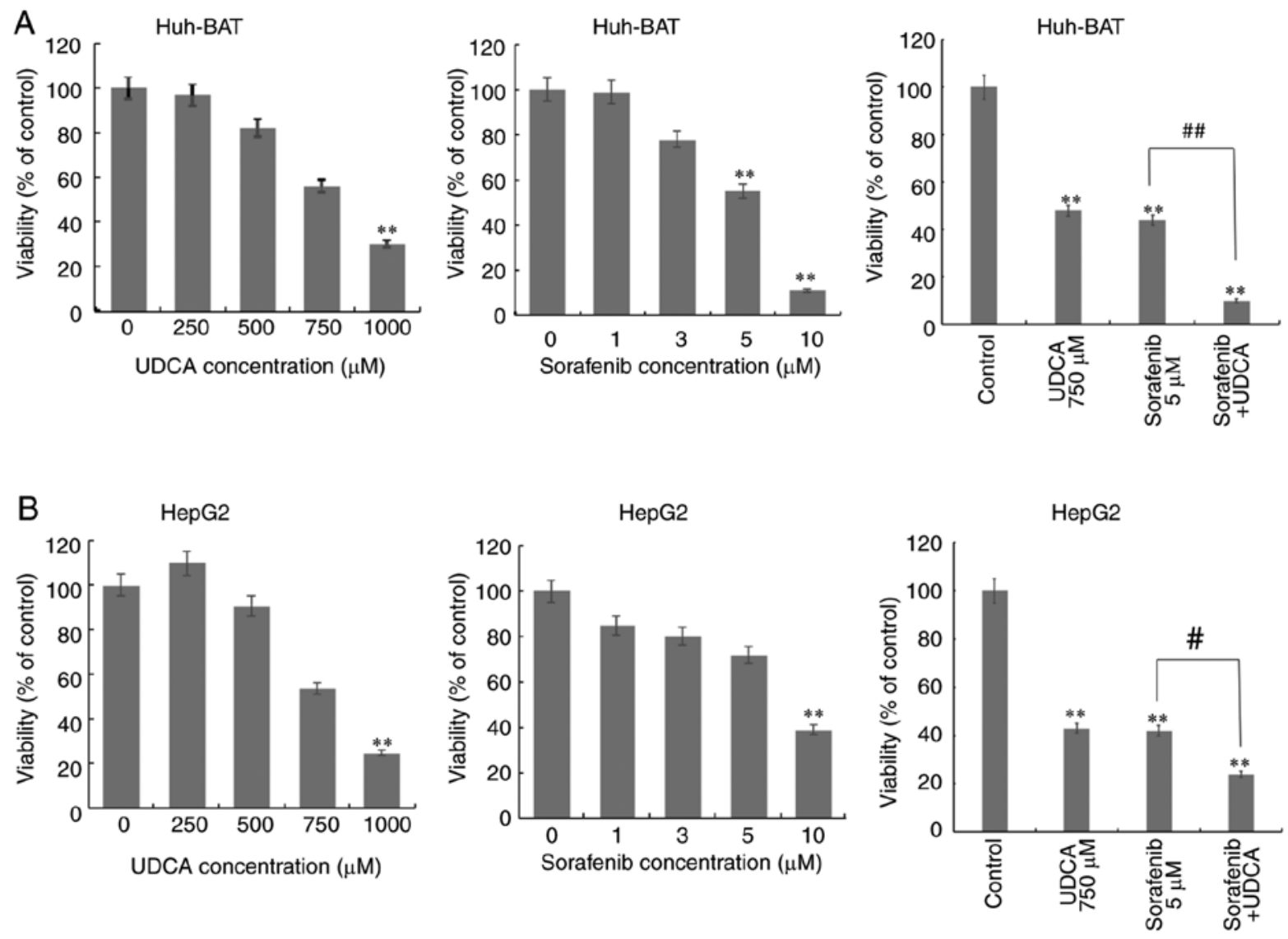

Figure 1. Sorafenib and UDCA inhibit hepatocellular carcinoma cell viability. (A) Huh-BAT and (B) HepG2 cells were treated for 48 h with different concentrations of sorafenib $(1,3,5$ and $10 \mu \mathrm{M})$, UDCA $(250,500,750$ and $1,000 \mu \mathrm{M})$, or co-treated with $5 \mu \mathrm{M}$ sorafenib and $750 \mu \mathrm{M}$ UDCA. Cell viability was measured using the MTT assay. ${ }^{* *} \mathrm{P}<0.01$, compared with control; ${ }^{\#} \mathrm{P}<0.05$ and ${ }^{\# \#} \mathrm{P}<0.01$ compared with sorafenib alone. UDCA, ursodeoxycholic acid.

Health, Bethesda, MD, USA). All western blot analyses were performed in triplicate.

Statistical analysis. All experiments were conducted in three independent repeats. Statistical analyses were performed using SPSS for Windows version 22.0 (IBM Corporation, Armonk, NY, USA), and one-way analysis of variance followed by Tukey's honest significant difference test. $\mathrm{P}<0.05$ was considered to indicate a statistically significant difference.

\section{Results}

Sorafenib and UDCA inhibit HCC cell viability. The cytotoxicity of co-treatment with sorafenib and UDCA was evaluated in Huh-BAT and HepG2 cells. HCC cells were treated with 0-10 $\mu \mathrm{M}$ sorafenib alone, $0-1,000 \mu \mathrm{M}$ UDCA alone, or $5 \mu \mathrm{M}$ sorafenib plus $750 \mu \mathrm{M}$ UDCA for $48 \mathrm{~h}$ (Fig. 1). Treatment with sorafenib or UDCA reduced the viability of Huh-BAT and HepG2 cells in a concentration-dependent manner, while co-treatment with both exhibited a significantly greater cytotoxicity compared with sorafenib alone.

Sorafenib and UDCA induce caspase-dependent apoptosis in HCC cells. Next, the present study evaluated whether co-treatment affected apoptosis of Huh-BAT and HepG2 cells. As illustrated in Fig. 2A, microscopy image analysis of the treated cells revealed that sorafenib, UDCA, and their co-treatment markedly altered cell morphology. These observations were confirmed by Annexin V/PI staining of cells treated with the indicated drugs (Fig. 2B and C). The ratio of apoptotic cells in the untreated control Huh-BAT group was 3\%, and this value increased to 15 and $9 \%$ following treatment with UDCA and sorafenib, respectively (Fig. 2C). Following co-treatment with sorafenib and UDCA, the \% of apoptotic cells increased to 18\% (Fig. 2C). For HepG2 cells, the ratio of apoptotic cells in the untreated control group was $6 \%$, which increased to $9 \%$ following treatment with either UDCA or sorafenib (Fig. 2C). Apoptosis increased significantly to $26 \%$ following co-treatment with both agents (Fig. 2C). These findings demonstrate that cell apoptosis was induced by sorafenib treatment, and this effect was enhanced when Huh-BAT and HepG2 cells were co-treated with sorafenib and UDCA.

To investigate the underlying mechanisms of the apoptosis effect, caspase- 3 and caspase- 9 expression was evaluated by western blotting. As illustrated in Fig. 2D, the results demonstrated that the expression levels of both the proteins and their cleaved forms were markedly increased in Huh-BAT and HepG2 cells co-treated with sorafenib and UDCA, compared with the cells incubated with sorafenib alone. These findings indicate that co-treatment induced Huh-BAT and HepG2 cell death via caspase-dependent apoptosis.

Sorafenib and UDCA suppress activation of STAT3 in a time- and concentration-dependent manner. Next, the effects of sorafenib 
A

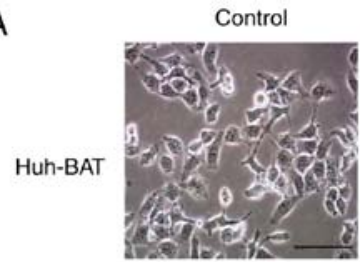

HepG2

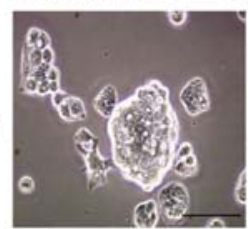

UDCA $750 \mu \mathrm{M}$
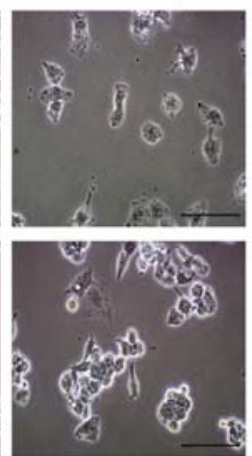

Sorafenib $5 \mu \mathrm{M}$
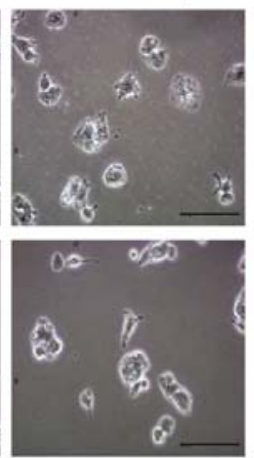

UDCA+Sorafenib
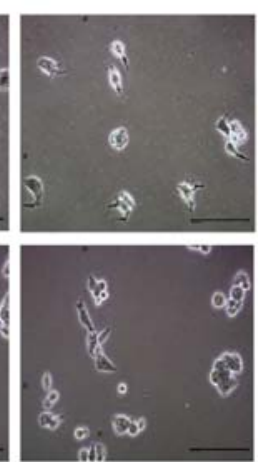

\section{B}

Control

UDCA $750 \mu \mathrm{M}$

Sorafenib $5 \mu \mathrm{M}$

UDCA+Sorafenib
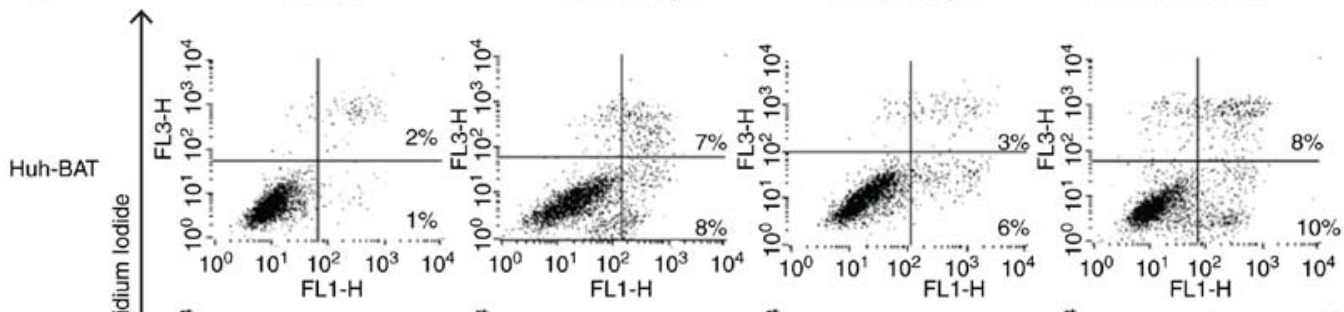

HepG2

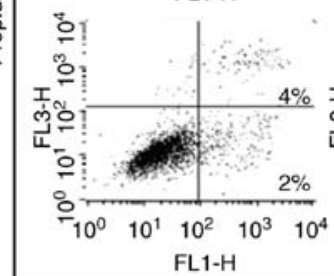

FL1-H
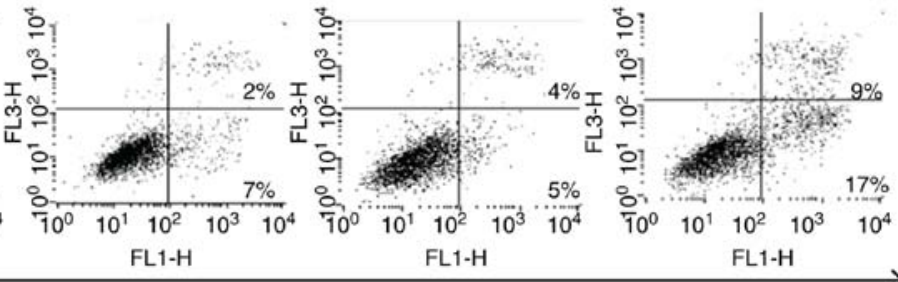

Annexin V-FITC
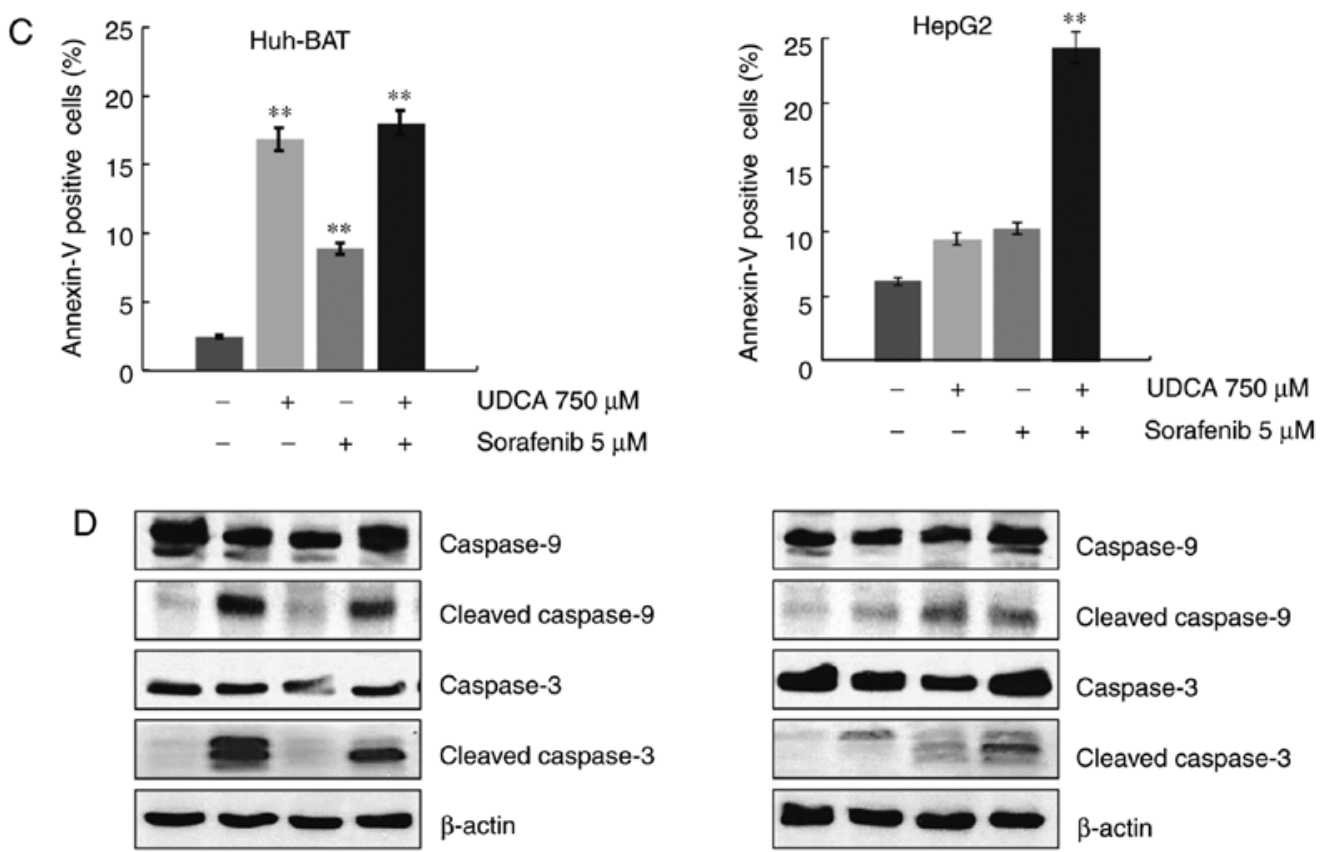

Figure 2. Sorafenib and UDCA induce caspase-dependent apoptosis in HCC cells. (A) Huh-BAT and HepG2 cells were treated with $5 \mu$ M sorafenib and $750 \mu \mathrm{M}$ UDCA for $48 \mathrm{~h}$. Cell morphology was observed by phase-contrast microscopy. Scale bar, $100 \mu \mathrm{m}$. (B) Representative plots and (C) quantification of flow cytometry analysis of cell apoptosis by Annexin V/PI staining. Cells were treated with $5 \mu \mathrm{M}$ sorafenib, $750 \mu \mathrm{M}$ UDCA, or both for $48 \mathrm{~h}$. The $\%$ of apoptotic cells was evaluated from three independent experiments. Data are presented as means \pm standard deviation. ${ }^{*} \mathrm{P}<0.05$ and ${ }^{* *} \mathrm{P}<0.01$ compared with control untreated cells. (D) Expression levels of cleaved caspase-3 and caspase-9 were determined by western blotting following 48 -h treatment with $5 \mu \mathrm{M}$ sorafenib, $750 \mu \mathrm{M}$ UDCA, or both. UDCA, ursodeoxycholic acid; FITC, fluorescein isothiocyanate.

and UDCA co-treatment were investigated on the phosphorylation of STAT3. Phosphorylation of STAT3 on Try705 decreased in both Huh-BAT and HepG2 cells treated with sorafenib or UDCA alone, both in a time- and concentration-dependent 
manner (Fig. 3A-D). The levels of phosphorylated STAT3 in both cell lines decreased following co-treatment with sorafenib and UDCA, compared with untreated cells (Fig. 3E). For immunofluorescence analysis, treatment with IL-6, an inflammatory factor that induces STAT3 phosphorylation on Tyr705, was used as a positive control. The results demonstrated that UDCA and sorafenib suppressed STAT3 phosphorylation (Fig. 3F). These findings suggest that co-treatment with these agents regulates phosphorylation of STAT3 on Try705 in both Huh-BAT and HepG2 cells.

Sorafenib and UDCA trigger ROS production and ERK activation in a time- and concentration-dependent manner. Intracellular ROS are important factors affecting the apoptosis of cancer cells in response to DNA damage. Thus, the generation of intercellular ROS was measured. Co-treatment with sorafenib and UDCA induced intracellular ROS generation in Huh-BAT and HepG2 cells, as detected by confocal microscopy (Fig. 4A and B). In the presence of sorafenib and UDCA alone, ROS generation increased compared with cells incubated with medium alone. The average fluorescent intensity appeared to increase compared with the cells incubated with sorafenib alone, although this difference was not significant.

Next, the phosphorylation of ERK in Huh-BAT and HepG2 cells was examined. Western blot analysis demonstrated that ERK was phosphorylated in both those cells treated with sorafenib or UDCA alone, in a time- and concentration-dependent manner (Fig. 4C-F). The levels of phosphorylated ERK in both cell lines increased following co-treatment with sorafenib and UDCA, compared with untreated cells (Fig. 4G). Compared with sorafenib alone, the levels of phospho-ERK were markedly increased following co-treatment in HepG2 cells, but this effect was not observed in Huh-Bat cells (Fig. 4G). These observations indicate that apoptosis induced by co-treatment with sorafenib and UDCA is associated with ROS production and is mediated by the phosphorylation of ERK in Huh-BAT and HepG2 cells.

ROS production and ERK activation are correlated with downregulation of STAT3 phosphorylation in Huh-BAT cells co-treated with sorafenib and UDCA. To investigate the relationship among ROS generation, ERK activation, and STAT3 dephosphorylation, Huh-BAT cells were treated with the ROS scavenger NAC, or the ERK inhibitor U0126 in the presence or absence of sorafenib, UDCA, or both. Firstly, cells were pretreated with NAC to assess the role of ROS. As presented in Fig. 5A, pretreatment with NAC effectively inhibited sorafenib- and UDCA-induced ROS production in Huh-BAT cells. Next, the roles of ERK and ROS in STAT3 inactivation by sorafenib and UDCA treatment were examined. Co-treatment with sorafenib and UDCA of Huh-BAT cells pretreated with U0126 or NAC significantly increased phospho-STAT3 levels and decreased those of phospho-ERK (Fig. 5B and C). These results indicate that ROS and ERK may have an important role in the suppression of STAT3 by sorafenib and UDCA treatment.

\section{Discussion}

In the present study, co-treatment with sorafenib and UDCA enhanced the effects of sorafenib on the inhibition of cell viability and the induction of apoptosis, via ROS-mediated activation of ERK and downregulation of STAT3 in HCC cells. To the best of our knowledge, this is the first study to demonstrate the synergistic effects of co-treatment with sorafenib and UDCA in HCC.

Several studies have investigated the role of STAT3 signaling in sorafenib-treated HCC cells, because STAT3 regulates the transcription of genes involved in cancer cell proliferation and apoptosis via the phosphoinositide 3-kinase (PI3K)/AKT signaling pathway (19). According to Blechacz et al (20), sorafenib inhibits STAT3 phosphorylation by downregulating protein tyrosine phosphatase non-receptor type 11 (PTPN11, also known as SHP-2) in cholangiocarcinoma cells. In addition, Gu et al (19) suggested that inhibition of STAT3 activity reduces tumor growth and metastasis in HCC, by partially suppressing the Raf/MAPK kinase (MEK)/ERK signaling pathway, independent of PTPN11. The present study also demonstrated that exposure to sorafenib suppressed STAT3 phosphorylation in HCC cells. However, the underlying mechanism mediating the antitumor actions of sorafenib were not consistent with those previously reported. The present results demonstrated that the reduced phosphorylation of STAT3 was rescued by U0126 in Huh-BAT cells, indicating that ERK activation participated in the inhibition of STAT3 in HCC cells. Although sorafenib is a well-known inhibitor of the Raf/MEK/ERK signaling pathway, the mode of action of sorafenib may vary depending on the response to stimuli under certain conditions, such as ROS generation.

Although ROS is produced during normal aerobic metabolism, excessive intracellular ROS generation causes oxidative stress and leads to cell damage, ultimately inducing apoptosis $(21,22)$. UDCA can stimulate lipid rafts and ROS production by acting on the cell membrane, thereby inducing apoptosis (23). Sorafenib has been reported to produce ROS (24). Co-treatment with these two pro-oxidants may have synergistic effects on ROS generation. Indeed, the present findings demonstrated that sorafenib and UDCA co-treatment increased ROS generation and ERK activation in Huh-BAT cells, while NAC reversed these effects.

Previous studies have demonstrated that ERK activation induces apoptosis under specific conditions, such as excessive ROS generation or oxidant scavenging failure (25). According to Zuo et al (26), alternol, an antitumor agent used in the treatment of osteosarcoma, increases ROS levels by activating ERK and subsequently inhibiting STAT3 phosphorylation. In addition, the reduced STAT3 phosphorylation by alternol was abrogated by pretreatment with NAC. These results are consistent with the current findings.

The present study was limited by the small number of cancer cell lines tested and the lack of in vivo experiments. Further studies are needed to investigate the effects of sorafenib and UDCA administration in vivo. In addition, not only the present study, but other UDCA-related preclinical studies use a relatively high concentration of UDCA as the $\mathrm{IC}_{50}$ value (27-29). It would be more beneficial to reduce the concentration of UDCA to protect normal cells, unless the lower concentration reduces its antitumor efficacy. In addition, it is necessary to identify whether reduced concentrations of sorafenib and UDCA would have the same cytotoxicity as the standard concentrations of sorafenib alone. 
A

Huh-BAT

UDCA $(\mu \mathrm{M})$ Sorafenib $(\mu \mathrm{M})$
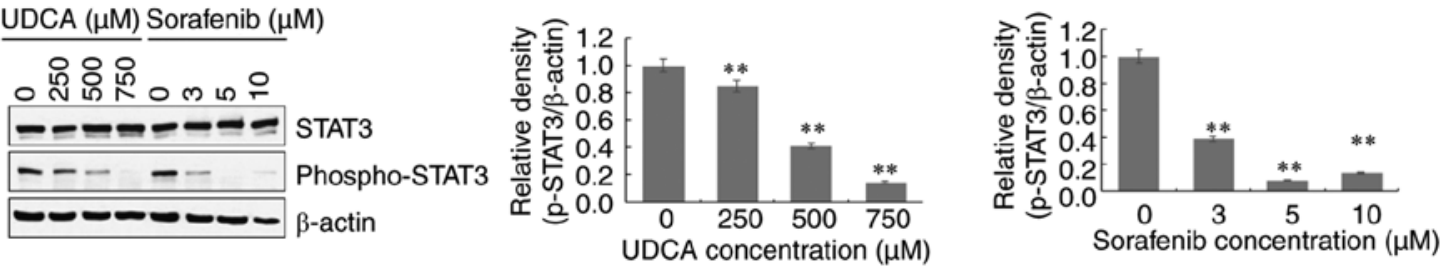

B

Huh-BAT

UDCA (h) Sorafenib (h)

02448720244872

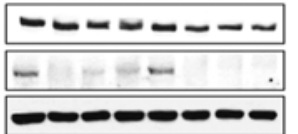

STAT3

Phospho-STAT3

$\beta$-actin

C

HepG2

UDCA $(\mu \mathrm{M})$ Sorafenib $(\mu \mathrm{M})$
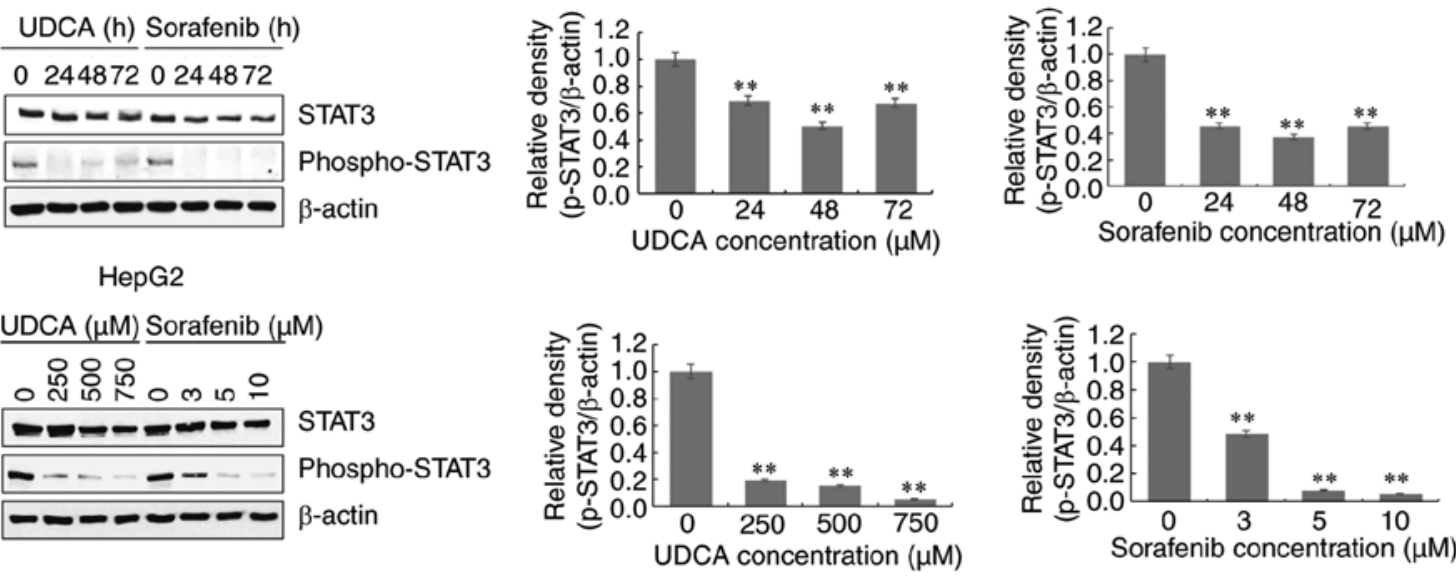

D

HepG2

\begin{tabular}{ll}
$\operatorname{UDCA}(\mathrm{h})$ & Sorafenib $(\mathrm{h})$ \\
\hline 0244872 & 0244872 \\
& STAT3 \\
\hline & P-actin
\end{tabular}
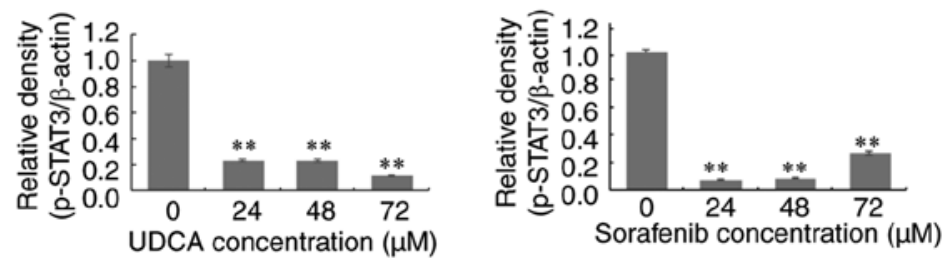

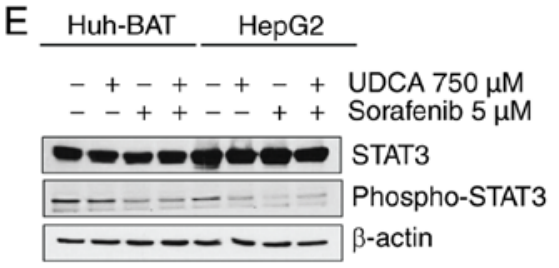

$\mathrm{F}$
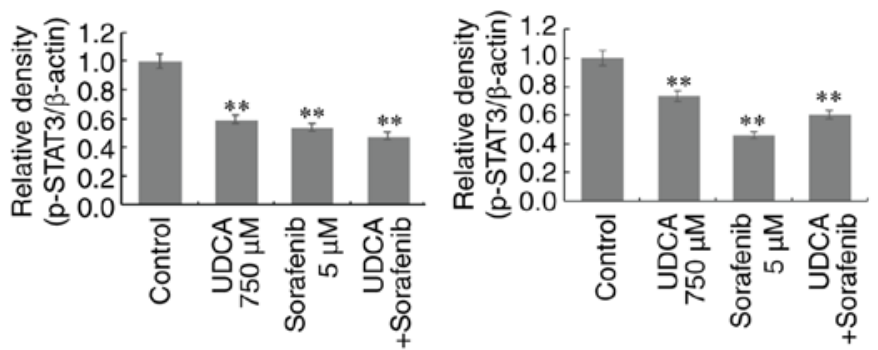

HepG2

DAPI
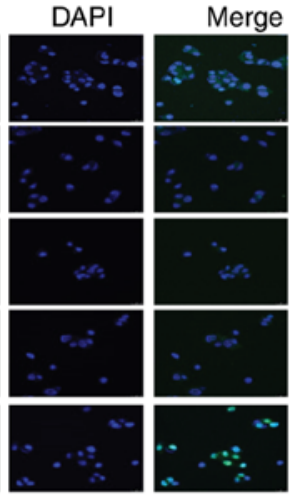

Figure 3. Sorafenib and UDCA suppress activation of STAT3 in a time- and concentration-dependent manner. (A) Huh-BAT cells were treated with various doses of UDCA or sorafenib for $48 \mathrm{~h}$. (B) Huh-BAT cells were treated with $750 \mu \mathrm{M}$ UDCA or $5 \mu \mathrm{M}$ sorafenib for various timepoints. (C) HepG2 cells were treated with various doses of UDCA or sorafenib for $48 \mathrm{~h}$. (D) HepG2 cells were treated with $750 \mu \mathrm{M}$ UDCA or $5 \mu \mathrm{M}$ sorafenib for various timepoints. (E) Cells were treated with $5 \mu \mathrm{M}$ sorafenib, $750 \mu \mathrm{M}$ UDCA or both for $48 \mathrm{~h}$. Protein expression levels of STAT3 and phospho-STAT3 were evaluated by western blotting. $\beta$-actin was used as a loading control. Representative blots and quantifications are shown for all panels. Data are presented as means \pm standard deviation. ${ }^{* *} \mathrm{P}<0.01$ compared with control untreated cells. (F) Huh-BAT and HepG2 cells were treated with $5 \mu \mathrm{M}$ sorafenib, $750 \mu \mathrm{M}$ UDCA, or both for $48 \mathrm{~h}$. Treatment with $50 \mathrm{ng} / \mathrm{ml}$ IL-6 for $30 \mathrm{~min}$ was used as a positive control for STAT3 activation. Following treatments, localization of phospho-STAT3 was evaluated using immunofluorescence. Magnification, x400. UDCA, ursodeoxycholic acid; STAT3, signal transducer and activator of transcription 3; IL-6, interkleukin-6. 

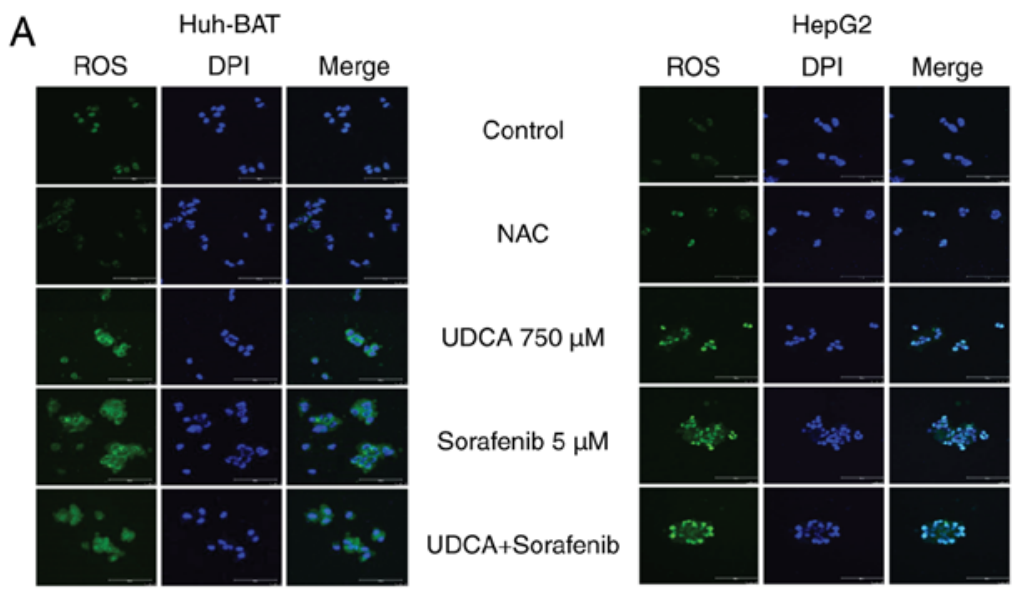

C Huh-BAT
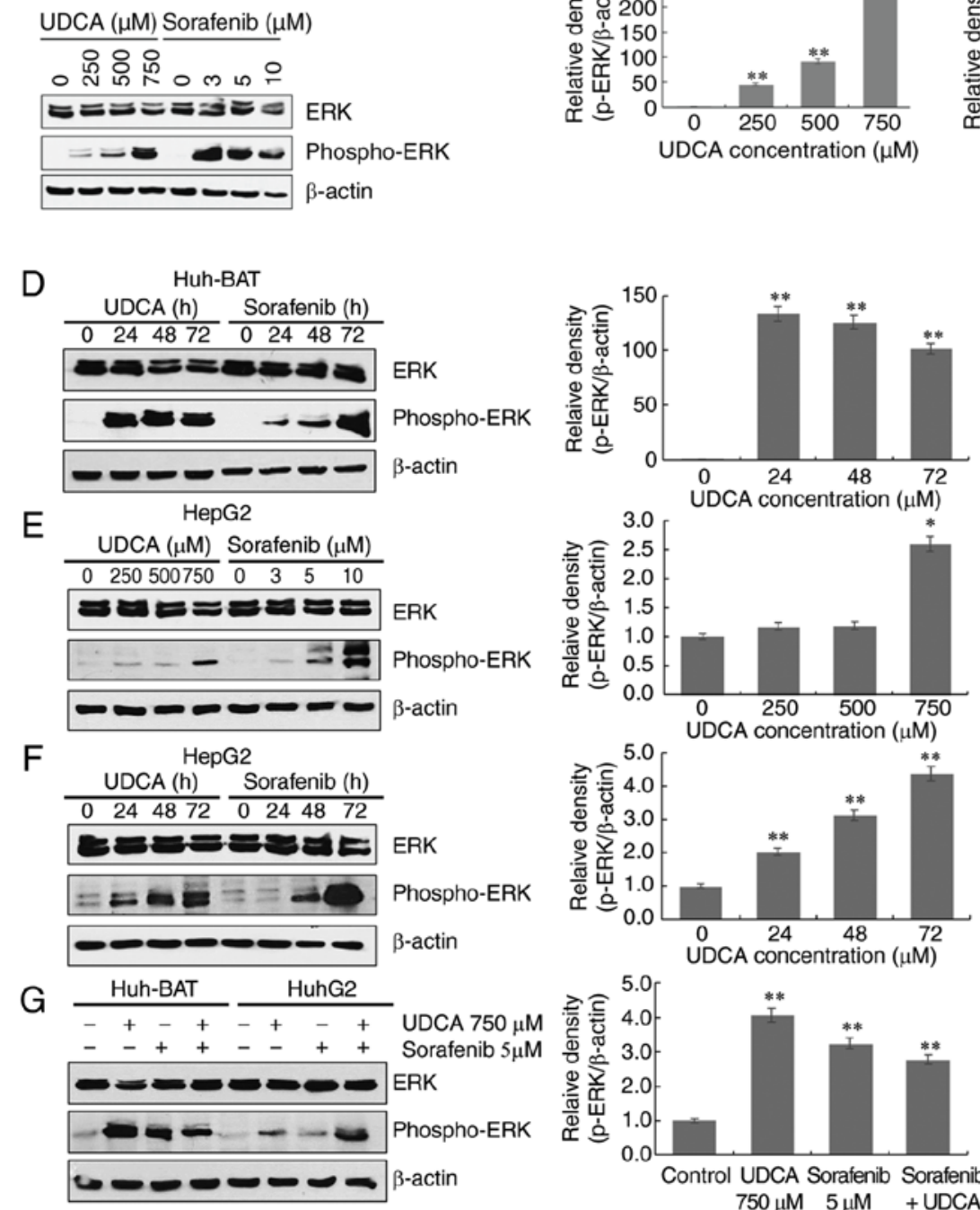
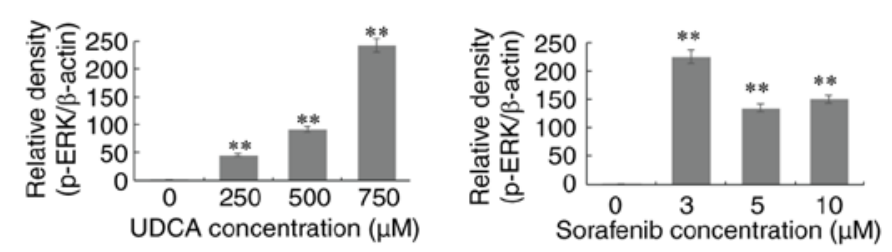
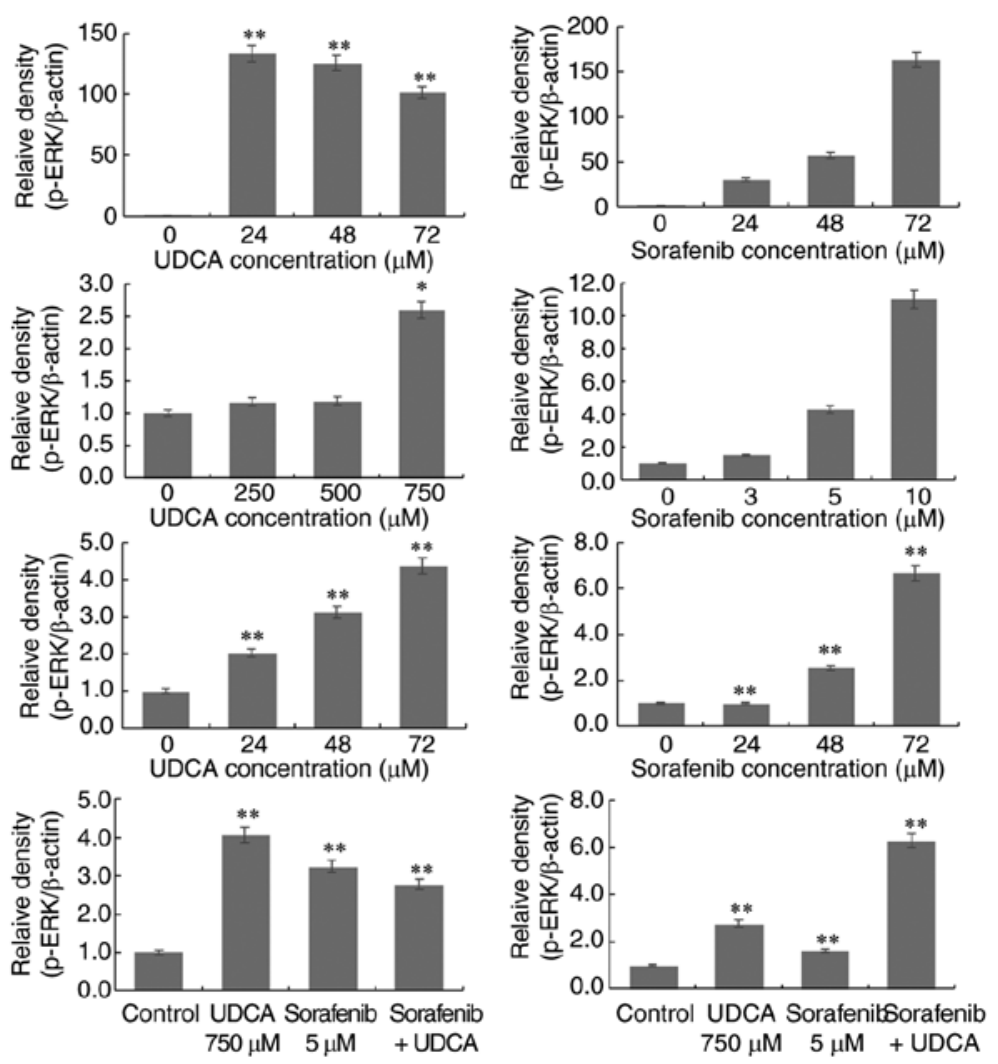

Figure 4. Sorafenib and UDCA trigger ROS production and activate ERK in a time- and concentration-dependent manner. (A) Huh-BAT and HepG2 cells were treated with $5 \mu \mathrm{M}$ sorafenib, $750 \mu \mathrm{M}$ UDCA, or both, or with $5 \mathrm{mM} \mathrm{NAC}$ for $48 \mathrm{~h}$. ROS generation was measured by confocal fluorescence microscopy. Magnification, x400. (B) Quantitative analysis of ROS generation. (C) Huh-BAT cells were treated with various doses of UDCA or sorafenib for $48 \mathrm{~h}$. (D) Huh-BAT cells were treated with $750 \mu \mathrm{M}$ UDCA or $5 \mu \mathrm{M}$ sorafenib for various timepoints. (E) HepG2 cells were treated with various doses of UDCA or sorafenib for $48 \mathrm{~h}$. (F) HepG2 cells were treated with $750 \mu \mathrm{M}$ UDCA or $5 \mu \mathrm{M}$ sorafenib for various timepoints. (G) Cells were treated with $5 \mu \mathrm{M}$ sorafenib, $750 \mu \mathrm{M}$ UDCA or both for $48 \mathrm{~h}$. Protein expression levels of ERK and phospho-ERK were evaluated by western blotting. $\beta$-actin was used as a loading control. Representative blots and quantifications are shown. Data are presented as means \pm standard deviation. ${ }^{*} \mathrm{P}<0.05$ and ${ }^{* *} \mathrm{P}<0.01$ compared with control untreated cells. UDCA, ursodeoxycholic acid; ROS, reactive oxygen species; ERK, extracellular signal-regulated kinase; NAC, N-acetyl cysteine.

Taken together, the present results demonstrated that sorafenib and UDCA suppressed the viability of HCC cells more effectively compared with sorafenib alone, and promoted apoptosis. Co-treatment with both agents inhibited STAT3 

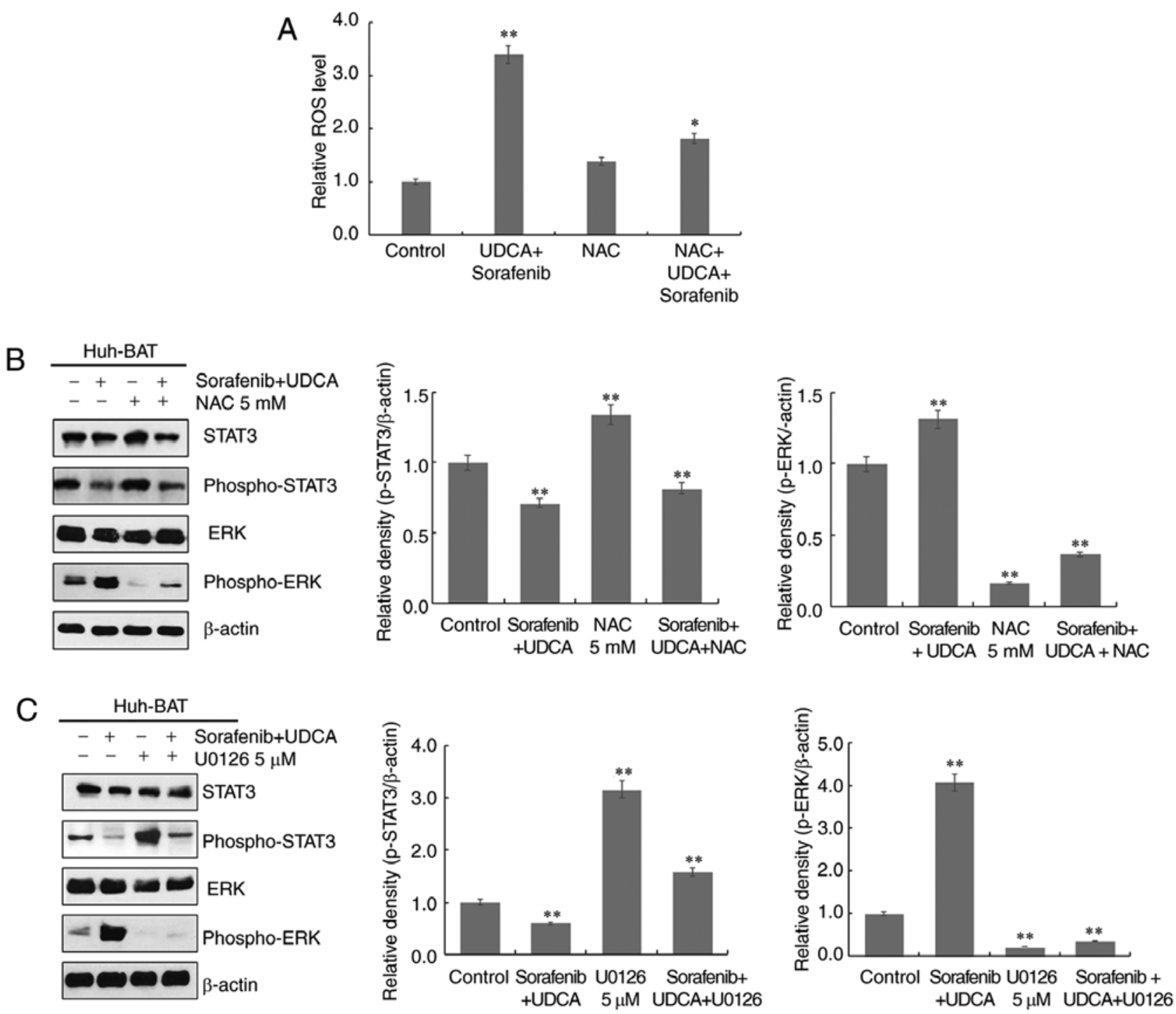

Figure 5. ROS production and ERK activation are associated with downregulation of STAT3 phosphorylation in Huh-BAT cells co-treated with sorafenib and UDCA. (A) Huh-BAT cells were co-treated with $5 \mu \mathrm{M}$ sorafenib and $750 \mu \mathrm{M}$ UDCA for $48 \mathrm{~h}$, followed by $5 \mathrm{mM}$ NAC. Following treatments, ROS intensity was measured using a fluorescence microplate reader. The relative ROS levels were normalized to the mean intensity of ROS in the control untreated cells (presented here as 1.0). (B) Huh-BAT cells were pretreated with $5 \mathrm{mM} \mathrm{NAC}$, or (C) pretreated with $5 \mu \mathrm{M}$ U0126, prior to $5 \mu \mathrm{M}$ sorafenib and $750 \mu \mathrm{M}$ UDCA treatments. Samples were immunoblotted with primary antibodies to STAT3, phospho-STAT3, ERK, and phospho-ERK. $\beta$-actin was used as a loading control. ${ }^{*} \mathrm{P}<0.05$ and ${ }^{* *} \mathrm{P}<0.01$ compared with control untreated cells. ROS, reactive oxygen species; ERK, extracellular signal-regulated kinase; STAT3, signal transducer and activator of transcription 3; UDCA, ursodeoxycholic acid; NAC, N-acetyl cysteine.

phosphorylation and activated ERK in a ROS-dependent manner. These findings suggest that the combination of sorafenib and UDCA may be a promising therapeutic strategy for patients with advanced HCC.

\section{Acknowledgements}

Not applicable.

\section{Funding}

This research was supported by the Daewoong Pharmaceutical Company (grant nos. 800-20150450 and 800-20150451), the Korea Health Technology R\&D Project through the Korea Health Industry Development Institute, funded by the Ministry of Health \& Welfare, Republic of Korea (grant no. HI16C1074) and the Seoul National
University Hospital Research Fund [grant no. 0420160300 (2016-1073)]

\section{Availability of data and materials}

The analyzed datasets generated during the study are available from the corresponding author on reasonable request.

\section{Authors' contributions}

SL performed the experiments, analyzed the data and wrote the manuscript. YYC critically revised the manuscript. EJC and SJY conceived and designed the study. JL and JY analyzed the data. YJK was involved in study design and manuscript revision and gave final approval of the version to be published. All authors approved the final version of the manuscript for publication. 


\section{Ethics approval and consent to participate}

Not applicable.

\section{Patient consent for publication}

Not applicable.

\section{Competing interests}

The authors declare that they have no competing interests.

\section{References}

1. Ferlay J, Soerjomataram I, Dikshit R, Eser S, Mathers C, Rebelo M, Parkin DM, Forman D and Bray F: Cancer incidence and mortality worldwide: Sources, methods and major patterns in GLOBOCAN 2012. Int J Cancer 136: E359-E386, 2015.

2. Llovet JM, Burroughs A and Bruix J: Hepatocellular carcinoma. Lancet 362: 1907-1917, 2003.

3. Gauthier A and Ho M: Role of sorafenib in the treatment of advanced hepatocellular carcinoma: An update. Hepatol Res 43: $147-154,2013$

4. Cheng AL, Kang YK, Chen Z, Tsao CJ, Qin SK, Kim JS, Luo R, Feng J, Ye S, Yang TS, et al: Efficacy and safety of sorafenib in patients in the Asia-Pacific region with advanced hepatocellular carcinoma: A phase III randomised, double-blind, placebo-controlled trial. Lancet Oncol 10: 25-34, 2009.

5. Llovet JM, Ricci S, Mazzaferro V, Hilgard P, Gane E, Blanc JF, de Oliveira AC, Santoro A, Raoul JL, Forner A, et al: Sorafenib in advanced hepatocellular carcinoma. N Engl J Med 359: 378-390, 2008.

6. Wilhelm SM, Carter C, Tang L, Wilkie D, McNabola A, Rong H, Chen C, Zhang X, Vincent P, McHugh M, et al: BAY 43-9006 exhibits broad spectrum oral antitumor activity and targets the RAF/MEK/ERK pathway and receptor tyrosine kinases involved in tumor progression and angiogenesis. Cancer Res 64: 7099-7109, 2004.

7. Almhanna K and Philip PA: Safety and efficacy of sorafenib in the treatment of hepatocellular carcinoma. Onco Targets Ther 2: 261-267, 2009.

8. Petrini I, Lencioni M, Ricasoli M, Iannopollo M, Orlandini C, Oliveri F, Bartolozzi C and Ricci S: Phase II trial of sorafenib in combination with 5-fluorouracil infusion in advanced hepatocellular carcinoma. Cancer Chemother Pharmacol 69: 773-780, 2012.

9. Wang L, Jia D, Duan F, Sun Z, Liu X, Zhou L, Sun L, Ren S, Ruan Y and Gu J: Combined anti-tumor effects of IFN- $\alpha$ and sorafenib on hepatocellular carcinoma in vitro and in vivo. Biochem Biophys Res Commun 422: 687-692, 2012.

10. Yang Y, Jiang H, Gao H, Kong J, Zhang P, Hu S, Shi B, Zhang P, Yao M and Li Z: The monoclonal antibody $\mathrm{CH} 12$ enhances the sorafenib-mediated growth inhibition of hepatocellular carcinoma xenografts expressing epidermal growth factor receptor variant III. Neoplasia 14: 509-518, 2012.

11. Greim H, Trülzsch D, Roboz J, Dressler K, Czygan P, Hutterer F, Schaffner F and Popper H: Mechanism of cholestasis. 5. Bile acids in normal rat livers and in those after bile duct ligation. Gastroenterology 63: 837-845, 1972.

12. Alberts DS, Martinez ME, Hess LM, Einspahr JG, Green SB Bhattacharyya AK, Guillen J, Krutzsch M, Batta AK, Salen G, et al: Phase III trial of ursodeoxycholic acid to prevent colorectal adenoma recurrence. J Natl Cancer Inst 97: 846-853, 2005.

13. Loddenkemper C, Keller S, Hanski ML, Cao M, Jahreis G, Stein H, Zeitz M and Hanski C: Prevention of colitis-associated carcinogenesis in a mouse model by diet supplementation with ursodeoxycholic acid. Int J Cancer 118: 2750-2757, 2006.
14. Sabharwal SS and Schumacker PT: Mitochondrial ROS in cancer: Initiators, amplifiers or an Achilles' heel? Nat Rev Cancer 14: 709-721, 2014.

15. Tan BJ and Chiu GN: Role of oxidative stress, endoplasmic reticulum stress and ERK activation in triptolide-induced apoptosis. Int J Oncol 42: 1605-1612, 2013.

16. Chen J, Wang J, Lin L, He L, Wu Y, Zhang L, Yi Z, Chen Y, Pang $X$ and Liu M: Inhibition of STAT3 signaling pathway by nitidine chloride suppressed the angiogenesis and growth of human gastric cancer. Mol Cancer Ther 11: 277-287, 2012.

17. Ryu K, Susa M, Choy E, Yang C, Hornicek FJ, Mankin HJ and Duan Z: Oleanane triterpenoid CDDO-Me induces apoptosis in multidrug resistant osteosarcoma cells through inhibition of Stat3 pathway. BMC Cancer 10: 187, 2010.

18. Wake MS and Watson CJ: STAT3 the oncogene-still eluding therapy? FEBS J 282: 2600-2611, 2015.

19. Gu FM, Li QL, Gao Q, Jiang JH, Huang XY, Pan JF, Fan J and Zhou J: Sorafenib inhibits growth and metastasis of hepatocellular carcinoma by blocking STAT3. World J Gastroenterol 17: 3922-3932, 2011

20. Blechacz BR, Smoot RL, Bronk SF, Werneburg NW, Sirica AE and Gores GJ: Sorafenib inhibits signal transducer and activator of transcription-3 signaling in cholangiocarcinoma cells by activating the phosphatase shatterproof 2. Hepatology 50: 1861-1870, 2009 .

21. Nordberg J and Arner ES: Reactive oxygen species, antioxidants, and the mammalian thioredoxin system. Free Radic Biol Med 31: $1287-1312,2001$

22. Circu ML and Aw TY: Reactive oxygen species, cellular redox systems, and apoptosis. Free Radic Biol Med 48: 749-762, 2010.

23. Lim SC, Duong HQ, Choi JE, Lee TB, Kang JH, Oh SH and Han SI: Lipid raft-dependent death receptor 5 (DR5) expression and activation are critical for ursodeoxycholic acid-induced apoptosis in gastric cancer cells. Carcinogenesis 32: 723-731, 2011.

24. Coriat R, Nicco C,Chereau C, Mir O, Alexandre J, Ropert S, Weill B, Chaussade S, Goldwasser F and Batteux F: Sorafenib-induced hepatocellular carcinoma cell death depends on reactive oxygen species production in vitro and in vivo. Mol Cancer Ther 11: 2284-2293, 2012.

25. Park BH, Lim JE, Jeon HG, Seo SI, Lee HM, Choi HY, Jeon SS and Jeong BC: Curcumin potentiates antitumor activity of cisplatin in bladder cancer cell lines via ROS-mediated activation of ERK1/2. Oncotarget 7: 63870-63886, 2016.

26. Zuo D, Zhou Z, Wang H, Zhang T, Zang J, Yin F, Sun W, Chen J, Duan L, Xu J, et al: Alternol, a natural compound, exerts an anti-tumour effect on osteosarcoma by modulating of STAT3 and ROS/MAPK signalling pathways. J Cell Mol Med 21: 208-221, 2017.

27. Pang L, Zhao X, Liu W, Deng J, Tan X and Qiu L: Anticancer effect of ursodeoxycholic acid in human oral squamous carcinoma HSC-3 cells through the caspases. Nutrients 7: 3200-3218, 2015.

28. Tsagarakis NJ, Drygiannakis I, Batistakis AG, Kolios G and Kouroumalis EA: A concentration-dependent effect of ursodeoxycholate on apoptosis and caspases activities of HepG2 hepatocellular carcinoma cells. Eur J Pharmacol 640: 1-7, 2010.

29. Krishna-Subramanian S, Hanski ML, Loddenkemper C, Choudhary B, Pagès G, Zeitz M and Hanski C: UDCA slows down intestinal cell proliferation by inducing high and sustained ERK phosphorylation. Int J Cancer 130: 2771-2782, 2012.

This work is licensed under a Creative Commons Attribution-NonCommercial-NoDerivatives 4.0 International (CC BY-NC-ND 4.0) License. 